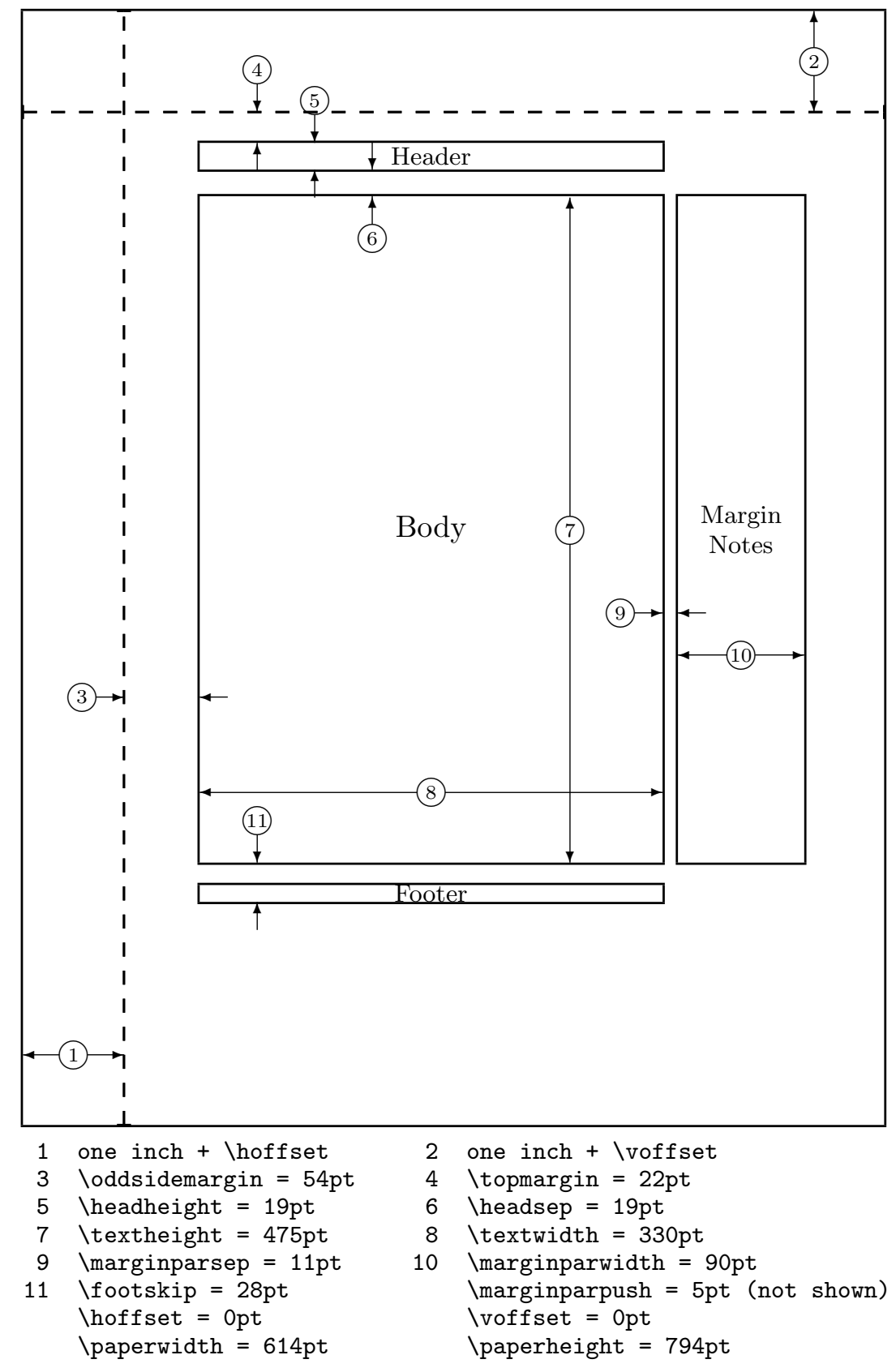




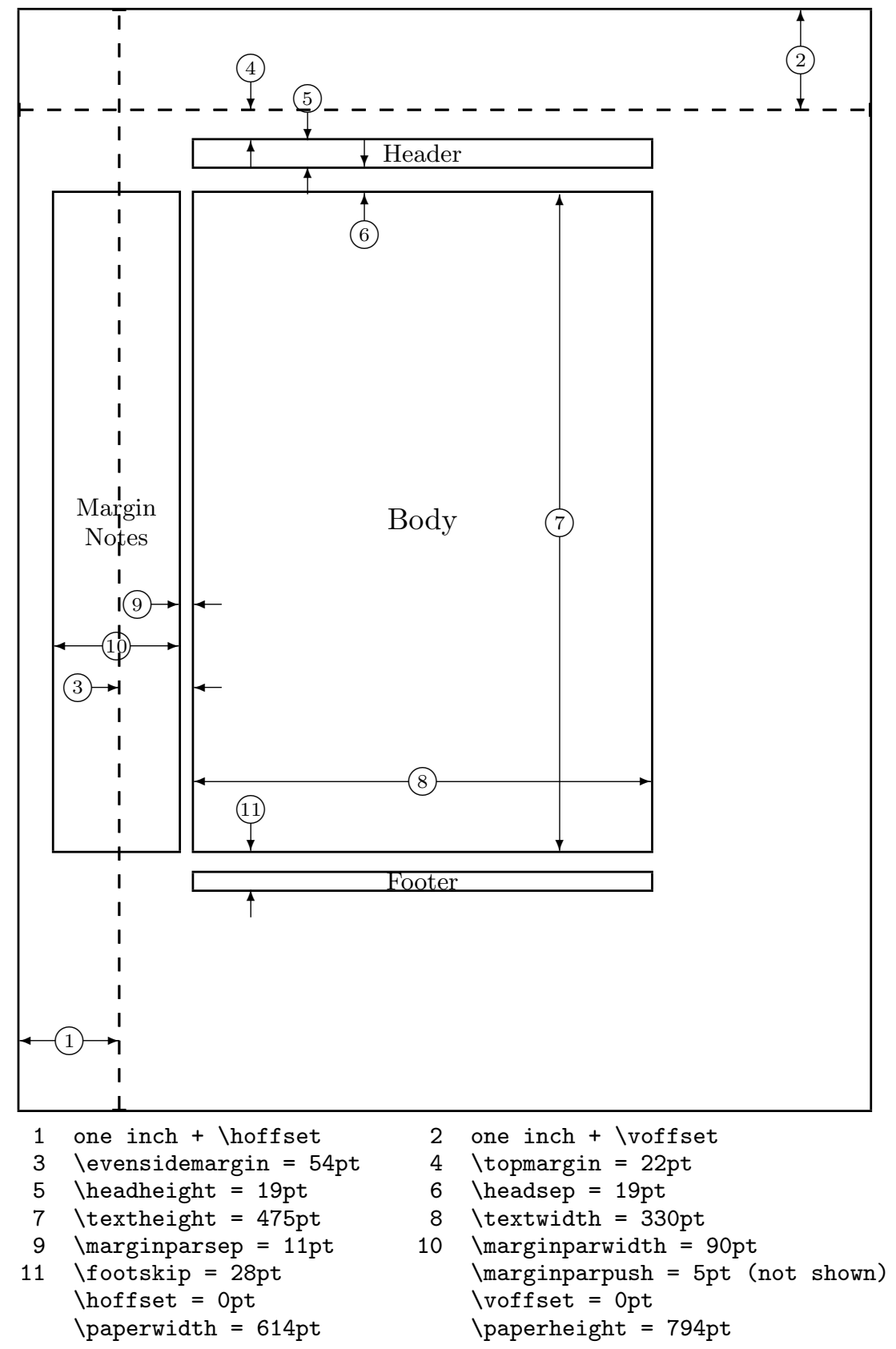




\title{
Partial orders related to the Hom-order and degenerations.
}

\section{Nils Nornes}

Norwegian University of Science and Technology, Department of Mathematical Sciences, N-7491 Trondheim, Norway.

E-mail address: nornes@math.ntnu.no

\begin{abstract}
Given a finite length module $M$ over a $K$-algebra $\Lambda$, an $n \times n$-matrix $A$ over $\Lambda$ induces a $K$-homomorphism $A_{M}$ : $M^{n} \rightarrow M^{n}$. We then define the relation $\leq_{n}$ by $M \leq_{n} N \Leftrightarrow \ell\left(\operatorname{coker} A_{M}\right) \leq \ell\left(\operatorname{coker} A_{N}\right)$.
\end{abstract}

We will show that $\leq_{n}$ is a partial order on the set of modules of length $d$ (modulo isomorphisms) if $n \geq d^{5}$.

The results presented in this paper are from the author's Master thesis. The author thanks S.O. Smalø for the help with both the thesis and this paper.

Throughout the paper let $\Lambda$ be an artin algebra with center $K$, and let $\bmod \Lambda$ denote the the category of finitely generated left $\Lambda$-modules. For a $\Lambda$-module $X, \ell(X)$ denotes the length of $X$ as a $K$-module. For a homomorphism $\phi, \operatorname{im} \phi$ denotes its image and coker $\phi$ denotes its cokernel.

For a natural number $d$, let $\operatorname{rep}_{d} \Lambda=\{X \in \bmod \Lambda \mid \ell(X)=d\}$. One can define several partial orders on $\operatorname{rep}_{d} \Lambda$ modulo isomorphisms (see [3]). Here we will look at the Hom-order and the quasiorders $\leq_{n}$, and investigate for which $n \leq_{n}$ is a partial order on $\operatorname{rep}_{d} \Lambda$.

Definition. The relation $\leq_{\text {Hom }}$ on $\operatorname{rep}_{d} \Lambda$ is defined by $M \leq_{\text {Hom }} N$ if $\ell\left(\operatorname{Hom}_{\Lambda}(X, M)\right) \leq \ell\left(\operatorname{Hom}_{\Lambda}(X, N)\right)$ for all $X \in \bmod \Lambda$. This relation is called the Hom-order.

2000 Mathematics Subject Classification: 16E30, 16G20, 16G60. 
We immediately see that $\leq_{\text {Hom }}$ is reflexive and transitive. That it is also antisymmetric was first shown by Auslander in [1], a result that will be stated here as Corollary 2 .

Let $\mathcal{M}_{n}(\Lambda)$ denote the ring of $n \times n$-matrices over $\Lambda$. For a matrix $A \in \mathcal{M}_{n}(\Lambda)$ and a $\Lambda$-module $M$, let $A_{M}: M^{n} \rightarrow M^{n}$ denote the $K$-homomorphism given by multiplying a column vector of elements from $M$ with the matrix $A$.

Definition. For a natural number $n$, the relation $\leq_{n}$ on $\operatorname{rep}_{d} \Lambda$ is defined by $M \leq_{n} N$ if $\ell\left(\operatorname{coker} A_{M}\right) \leq \ell\left(\operatorname{coker} A_{N}\right)$ for all $A \in \mathcal{M}_{n}(\Lambda)$.

Again it is obvious that this relation is reflexive and transitive, but it is not in general antisymmetric. We have that $\ell\left(\right.$ coker $\left.A_{M}\right)=$ $\ell\left(\operatorname{Hom}_{\Lambda}\left(\Lambda^{n} / \Lambda^{n} A, M\right)\right)$, where $\Lambda^{n} A$ denotes the image of the $\Lambda$ - endomorphism induced by matrix multiplication from the right with $A$. Hence, $M \leq_{\text {Hom }} N$ implies $M \leq_{n} N$. We also have that $M \leq_{n+1} N$ implies $M \leq_{n} N$ for any $n$. It follows that if $\leq_{i}$ is a partial order, then so is $\leq_{n}$ for all $n \geq i$. If $K$ is an algebraically closed field, then we have by Hilbert's Basis Theorem that there must exist a minimal $n$ such that $\leq_{n}$ is equivalent to $\leq_{\text {Hom }}$ on $\operatorname{rep}_{d}(\Lambda)$. However, the Basis Theorem does not give any clue about how large this $n$ is, and there is no known procedure for finding it. It is also not known if this is the same as the minimal $n$ that makes $\leq_{n}$ a partial order.

This latter $n$ we can at least give a bound on. The following result was stated without proof in [3].

Theorem 1. For $n \geq d^{5}$ the relation $\leq_{n}$ is a partial order on $\operatorname{rep}_{d} \Lambda$.

Proof. We need to show that $\leq_{n}$ is antisymmetric when $n \geq d^{5}$. Let $M$ and $N$ be non-isomorphic $\Lambda$-modules of length $d$. First we want to show that there exists a $\Lambda$-module $X$ with $\ell\left(\operatorname{Hom}_{\Lambda}(X, M)\right) \neq$ $\ell\left(\operatorname{Hom}_{\Lambda}(X, N)\right)$ and $\ell(X) \leq d^{3}$. If $M$ and $N$ have any nonzero common direct summands, we can cancel them, so we assume they have none.

Let $\left\{f_{1}, f_{2}, \ldots, f_{m}\right\}$ be a generating set for $\operatorname{Hom}_{\Lambda}(M, N)$ as a Kmodule. Letting $C=\operatorname{coker}\left(\left(f_{1}, f_{2}, \ldots, f_{m}\right)^{t r}\right)$ we have an exact sequence

$$
M \stackrel{\left(f_{1}, f_{2}, \ldots, f_{m}\right)^{t r}}{\rightarrow} N^{m} \rightarrow C \rightarrow 0 .
$$


We have $\operatorname{Hom}_{\Lambda}(M, N) \subseteq \operatorname{Hom}_{K}(M, N)$ as a K-module, so $m \leq d^{2}$. Hence $\ell(C) \leq \ell\left(N^{m}\right) \leq \bar{d}^{3}$. Applying $\operatorname{Hom}_{\Lambda}(-, M)$ and $\operatorname{Hom}_{\Lambda}(-, N)$ to the above sequence we get

$0 \rightarrow \operatorname{Hom}_{\Lambda}(C, M) \rightarrow \operatorname{Hom}_{\Lambda}\left(N^{m}, M\right) \stackrel{\operatorname{Hom}_{\Lambda}\left(\left(f_{1}, f_{2}, \ldots, f_{m}\right)^{t r}, M\right)}{\rightarrow} \operatorname{Hom}_{\Lambda}(M, M)$

and

$0 \rightarrow \operatorname{Hom}_{\Lambda}(C, N) \rightarrow \operatorname{Hom}_{\Lambda}\left(N^{m}, N\right) \stackrel{\operatorname{Hom}_{\Lambda}\left(\left(f_{1}, f_{2}, \ldots, f_{m}\right)^{t r}, N\right)}{\rightarrow} \operatorname{Hom}_{\Lambda}(M, N)$.

$\operatorname{Hom}_{\Lambda}\left(\left(f_{1}, f_{2}, \ldots, f_{m}\right)^{t r}, N\right)$ is an epimorphism by construction. Now we assume

$$
\begin{gathered}
\ell\left(\operatorname{Hom}_{\Lambda}(C, M)\right)=\ell\left(\operatorname{Hom}_{\Lambda}(C, N)\right), \\
\ell\left(\operatorname{Hom}_{\Lambda}(M, M)\right)=\ell\left(\operatorname{Hom}_{\Lambda}(M, N)\right), \\
\ell\left(\operatorname{Hom}_{\Lambda}(N, M)\right)=\ell\left(\operatorname{Hom}_{\Lambda}(N, N)\right) .
\end{gathered}
$$

From the sequences (1) and (2) we then get

$$
\begin{aligned}
& \ell\left(\operatorname{im}_{\operatorname{Hom}_{\Lambda}}\left(\left(f_{1}, f_{2}, \ldots, f_{m}\right)^{t r}, M\right)\right) \\
= & \ell\left(\operatorname{Hom}_{\Lambda}\left(N^{m}, M\right)\right)-\ell\left(\operatorname{Hom}_{\Lambda}(C, M)\right) \\
= & \ell\left(\operatorname{Hom}_{\Lambda}\left(N^{m}, N\right)\right)-\ell\left(\operatorname{Hom}_{\Lambda}(C, N)\right. \\
= & \ell\left(\operatorname{Hom}_{\Lambda}(M, N)=\ell\left(\operatorname{Hom}_{\Lambda}(M, M)\right)\right.
\end{aligned}
$$

and hence $\operatorname{Hom}_{\Lambda}\left(\left(f_{1}, f_{2}, \ldots, f_{m}\right)^{t r}, M\right)$ is an epimorphism.

In particular the identity on $M$ factors through $\left(f_{1}, f_{2}, \ldots, f_{m}\right)^{t r}$, so $\left(f_{1}, f_{2}, \ldots, f_{m}\right)^{t r}$ must be a split monomorphism. Then $M$ and $N$ must have a common nonzero direct summand, which is a contradiction. Consequently one of the assumptions (3), (4) and (5) must fail and we have found the desired $X$.

We now show that there exists a $d^{5} \times d^{5}$-matrix $A$ with entries from $\Lambda$ such that $\ell\left(\operatorname{coker} A_{M}\right) \neq \ell\left(\operatorname{coker} A_{N}\right)$. If ann $M \neq \operatorname{ann} N$ there is a $\lambda \in \Lambda$ with $\ell\left(\operatorname{coker} \lambda_{M}\right) \neq \ell\left(\operatorname{coker} \lambda_{N}\right)$, so we assume $\operatorname{ann} M=\operatorname{ann} N$. Let $\Gamma=\Lambda / \operatorname{ann} M$. We have $\operatorname{Hom}_{\Lambda}(X, M) \simeq$ 
$\operatorname{Hom}_{\Lambda}(X /(\operatorname{ann} M) X, M)$, so we may assume that $X$ is annihilated by ann $M$. Then we have $\operatorname{Hom}_{\Lambda}(X, M) \simeq \operatorname{Hom}_{\Gamma}(X, M)$.

We have $\Gamma \subseteq \operatorname{End}_{K} M$ and thus $\ell(\Gamma) \leq d^{2}$. We can now make a free resolution of $X$ as a $\Gamma$-module:

$$
\Gamma^{d^{5}} \rightarrow \Gamma^{d^{3}} \rightarrow X \rightarrow 0
$$

and from this we get the exact sequence

$$
\Gamma^{d^{5}} \stackrel{A}{\rightarrow} \Gamma^{d^{5}} \stackrel{g}{\rightarrow} X \oplus \Gamma^{\left(d^{5}-d^{3}\right)} \rightarrow 0
$$

Applying $\operatorname{Hom}_{\Gamma}(-, M)$ and $\operatorname{Hom}_{\Gamma}(-, N)$ to this sequence we get

$$
0 \rightarrow \operatorname{Hom}_{\Gamma}(X, M) \oplus M^{d^{5}-d^{3}} \rightarrow M^{d^{5}} \stackrel{A_{M^{I}}}{\rightarrow} M^{d^{5}} \rightarrow \operatorname{coker} A_{M} \rightarrow 0
$$

and

$$
0 \rightarrow \operatorname{Hom}_{\Gamma}(X, N) \oplus N^{d^{5}-d^{3}} \rightarrow N^{d^{5}} \stackrel{A_{N}}{\rightarrow} N^{d^{5}} \rightarrow \operatorname{coker} A_{N} \rightarrow 0 .
$$

From this we get

$$
\begin{aligned}
\ell\left(\operatorname{coker} A_{M}\right) & =\ell\left(\operatorname{Hom}_{\Gamma}(X, M)\right)+\ell\left(M^{d^{5}-d^{3}}\right) \\
\ell\left(\operatorname{coker} A_{N}\right) & =\ell\left(\operatorname{Hom}_{\Gamma}(X, N)\right)+\ell\left(N^{d^{5}-d^{3}}\right)
\end{aligned}
$$

Since $\ell\left(\operatorname{Hom}_{\Gamma}(X, M)\right) \neq \ell\left(\operatorname{Hom}_{\Gamma}(X, N)\right)$ we then have $\ell\left(\right.$ coker $\left.A_{M}\right) \neq$ $\ell\left(\operatorname{coker} A_{N}\right)$.

Corollary 2. If $M$ and $N$ are non-isomorphic modules in $\bmod \Lambda$, then there exists a module $X$ in $\bmod \Lambda$ such that $\ell\left(\operatorname{Hom}_{\Lambda}(M, X)\right) \neq$ $\ell\left(\operatorname{Hom}_{\Lambda}(N, X)\right)$.

For algebras of finite representation type one can get a better estimate of $n$ by looking at the minimal projective presentations of the indecomposable modules. Here one can find an $n$ such that $\leq_{n}$ is a partial order on $\operatorname{rep}_{d} \Lambda$ for all $d$. (For background information on algebras of finite type see chapters VI and VIII.5 in [2].)

For each indecomposable $\Lambda$-module $X$ let

$$
P_{X}^{\prime} \rightarrow P_{X} \rightarrow X \rightarrow 0
$$

be its minimal projective presentation.

Proposition 3. If for all indecomposable modules $X$ in $\bmod \Lambda$ neither $P_{X}$ nor $P_{X}^{\prime}$ have more than $n$ copies of any indecomposable module as a direct summand, then $\leq_{n}$ is a partial order on $\operatorname{rep}_{d} \Lambda$ for any natural number $d$. 
Proof. Let $M$ and $N$ be non-isomorphic modules. There exists an indecomposable module $X$ such that $\ell\left(\operatorname{Hom}_{\Lambda}(X, M)\right) \neq \ell\left(\operatorname{Hom}_{\Lambda}(X, N)\right)$. From the minimal projective presentation of $X$ we can make an exact sequence

$$
\Lambda^{n} \rightarrow \Lambda^{n} \rightarrow X \oplus P \rightarrow 0
$$

where $P$ is projective, and from there we proceed as in the proof of Theorem 1.

If $\Lambda$ is of infinite representation type, then for any $n$ there exists an indecomposable module $X$ such that $P_{X}$ is not a direct summand of $\Lambda^{n}$. Let

$$
0 \rightarrow D \operatorname{Tr} X \rightarrow Y \rightarrow X \rightarrow 0
$$

be the almost split sequence ending in $X$ and let $Z=X \oplus D \operatorname{Tr} X$. If there is a matrix $A \in \mathcal{M}_{n}(\Lambda)$ such that $\ell\left(\operatorname{coker} A_{Z}\right) \neq \ell\left(\operatorname{coker} A_{Y}\right)$, we get that $X$ is a direct summand in $\Lambda^{n} / \Lambda^{n} A$, which leads to a contradiction. We therefore have $\ell\left(\operatorname{coker} A_{Z}\right)=\ell\left(\operatorname{coker} A_{Y}\right)$ for all $n \times n$-matrices $A$. Hence it is impossible to find an $n$ that makes $\leq_{n}$ a partial order for all $\operatorname{rep}_{d} \Lambda$ in this case.

The hereditary algebras of finite type can be described as path algebras over a particular class of quivers, the Dynkin quivers (see III.1 and VIII.5 in [2]). Applying Proposition 3 to these, we get the following result:

Proposition 4. Let $k$ be field and $Q$ be a quiver. Then we have the following.

(1) If the underlying graph of $Q$ is $A_{n}$, then $\leq_{1}$ is a partial order on $\operatorname{rep}_{d} k Q$ for any $d$.

(2) If the underlying graph of $Q$ is $D_{n}$, then $\leq_{2}$ is a partial order on $\operatorname{rep}_{d} k Q$ for any $d$. Depending on the orientation of $Q$, we may also have that $\leq_{1}$ is a partial order on $\operatorname{rep}_{d} k Q$ for any $d$.

(3) If the underlying graph of $Q$ is $E_{6}$, then $\leq_{3}$ is a partial order on $\operatorname{rep}_{d} k Q$ for any $d$. Depending on the orientation of $Q$, we may also have that $\leq_{2}$ is a partial order on $\operatorname{rep}_{d} k Q$ for any $d$.

(4) If the underlying graph of $Q$ is $E_{7}$, then $\leq_{4}$ is a partial order on $\operatorname{rep}_{d} k Q$ for any $d$. Depending on the orientation of $Q$, we may also have that $\leq_{3}$ and $\leq_{2}$ are partial orders on $\operatorname{rep}_{d} k Q$ for any $d$.

(5) If the underlying graph of $Q$ is $E_{8}$, then $\leq_{6}$ is a partial order on $\operatorname{rep}_{d} k Q$ for any $d$. Depending on the orientation of $Q$, 
we may also have that $\leq_{5}, \leq_{4}$ and $\leq_{3}$ are partial orders on $\operatorname{rep}_{d} k Q$ for any $d$.

For the cases in Proposition $4, \leq_{n}$ is a partial order if and only if it is equivalent to $\leq_{\text {Hom }}$. Whether this holds in $\operatorname{rep}_{d} \Lambda$ for any algebra $\Lambda$ and any natural number $d$ is an open problem.

\section{References}

[1] M. Auslander: Representation theory of finite dimensional algebras. Contemp. Math. 13, (1982), pp. 27-39.

[2] M. Auslander, I. Reiten and S. O. Smalø: Representation Theory of Artin Algebras. Cambridge studies in advanced mathematics 36, (1995).

[3] S. O. Smalø: Degenerations of Representations of Associative Algebras. Milan Journal of Math. 76, nr. 1, (2008), pp. 135-164. 